\title{
Aromatherapy in dementia
}

\author{
Clive Holmes \& Clive Ballard
}

\begin{abstract}
The use of plant essential oils in aromatherapy has been recorded for thousands of years, but until recently there was very little formal evidence regarding the use of these treatments in people with dementia. Over the past few years a number of clinical trials have compared aromatherapy, principally using either lavender (Lavandula angustifolia or Lavandula officinalis) or lemon balm (Melissa officinalis), with inactive treatment. All of these studies demonstrated a significant impact on behavioural problems in patients with dementia, with negligible side-effects. However, there is still not sufficient evidence to recommend widespread use in clinical practice and the key question of whether these treatments can provide a viable alternative to existing pharmacological agents needs to be addressed.
\end{abstract}

Aromatherapy is the use of pure essential oils, highly fragrant essences extracted from plants by distillation (Price \& Price, 2000: pp. 7-22). They are most commonly used in oil burners, soaked into pillows and tissues or massaged into the skin. The application of plant essential oils in aromatherapy has been recorded for thousands of years, and knowledge of the distillation and application of essential oils to improve general well-being and specific health problems was introduced into Europe as early as the 10th century. Several plant species, including lemon balm, lavender, chamomile, bergamot, neroli and valerian, have been used in medical herbalism specifically for their perceived beneficial effects on mental health problems such as anxiety and depression (Kite et al, 1998). In the context of dementing illness, lavender and lemon balm are of particular interest on account of their supposed sedative and/or cognitive enhancing properties and, more recently, because of emerging supportive neurochemical and clinical studies.

\section{Mechanisms of action of aromatic essential oils (Box 1) \\ Psychological aspects}

Aromatic oils are, by definition, fragrant. A number of psychological responses to fragrant odours are possible, but they include the individual's perception of whether a particular odour is pleasant or unpleasant and the individual's past associations with that odour. These variable individual psychological effects of odours are important since they are likely to influence treatment outcomes.

In most circumstances a high concentration of most odours, even when in small quantities they may be considered pleasant, can be considered unpleasant. Thus, an early study (Ludvigson \& Rottman, 1989) of healthy people using high levels of lavender (compared with no odour) found that cognitive functioning, particularly for arithmetic, was reduced by the lavender. However, a later study (Degel \& Koster, 1999) comparing the effects of lavender and jasmine on arithmetical and concentration tasks, using imperceptible or unnoticed levels of odour, found that lavender reduced the number of

Box 1 Psychological and neurochemical aspects of treatment response

Psychological aspects

- The individual's perception of the pleasantness of an odour

- The individual's past association with an odour

Neurochemical effects

- Inhibition of glutamate binding

- GABA augmentation

- Acetylcholine receptor binding

Clive Holmes is a senior lecturer in old age psychiatry at the University of Southampton (Memory Assessment and Research Unit, Moorgreen Hospital, Botley Road, West End, Southampton SO30 3JB, UK. E-mail: ch4@soton.acuk). Dr Holmes trained in psychiatry at both Guy's and the Maudsley Hospitals, London, and he has studied the neurochemistry and the genetics of Alzheimer's disease at the Institute of Neurology, London, and the Institute of Psychiatry, London, respectively. Clive Ballard is Professor of Old Age Psychiatry at the Institute of Ageing and Health at the University of Newcastle, UK. Professor Ballard trained in psychiatry at Birmingham University and his main interests are in the aetiology and treatment of behavioural and psychological symptoms of dementia. 
errors made in the arithmetical and concentration tasks (compared with the control and jasmine groups). This suggests that improvements are more likely to be seen if the aromatic oil is not in high concentrations that can be perceived as being unpleasant.

Individual experience of an odour may also affect response. The marked association of odours with emotional response is due to the prominence of afferent links from the olfactory bulb to the amygdala, where emotional significance is attached to incoming stimuli (Clark \& Boutros, 1999: pp. 143157). Consequently, study participants for whom a particular odour has strong negative (or positive) associations may be expected to introduce further inter-individual variability in outcome measures.

\section{Biological aspects}

Unlike psychological mechanisms, the pharmacological mechanism for aromatherapy is not thought to involve any perception of the odour. Here the effects are thought to be due to the compounds entering the body and acting directly on the brain, i.e. via the bloodstream by absorption through the lungs or olfactory mucosa. That aromatherapy might have its effects in the absence of any psychological perception of the smell is important, since many people with dementia may be anosmic because of the early loss of olfactory neurons (Vance, 1999).

\section{Preclinical studies}

The biological effects of aromatherapy may, in part, be due to general properties of aromatic oils, although the molecular properties of the specific aromatherapy ingredient used may also be important.

The main terpenoid component of lavender oil is linalool. Linalool is lipophilic and, as might be expected given the highly lipophilic nature of the brain, the components are readily transported across the blood-brain barrier. Linalool has been shown to inhibit glutamate binding in rats (Elisabetsky et al, 1995) and inhaled lavender oil to reduce electroshock-induced convulsions in mice, which suggests that there may also be a $\gamma$-amiobutyric acid (GABA) augmentation of action (Yamada et al, 1994). Further evidence for this mechanism comes from the finding of a potentiation of $\mathrm{GABA}_{\mathrm{A}}$ receptors expressed in Xenopus oocytes by lavender oil components (Aoshima \& Hamamoto, 1999). The main terpene components of lemon balm are citronellal, caryophylline, neral, garaniol, geranyl acetate and citronallal, which are also highly lipophilic. Lemon balm has also been shown to have relevant bioactivity, probably related to its terpene constituents. For example, it has been shown in some studies to displace nicotine from nicotinic acetylcholine receptors and scopolamine from muscarinic receptors. There is, however, considerable variation in cholinoreceptor interactions, probably because extracts themselves vary (Wake et al, 2000).

Animal behavioural studies further support a pharmacological action. For example, Buchbauer et al (1993) found that a 78\% decrease in the motility of mice $(92 \%$ if they were first agitated with a caffeine injection) resulted from inhalation of ambient lavender oil for $1 \mathrm{~h}$.

\section{Clinical studies}

\section{Individual case reports}

The use of aromatherapy oils for the treatment of behavioural problems in people with dementia is largely unexplored in placebo-controlled or casecontrol studies. However, a large number of small, uncontrolled case studies show efficacy (Henry et al, 1994; West \& Brockman, 1994; Hardy et al, 1995; Wolfe \& Herzberg, 1996; Brooker et al, 1997; MacMahon \& Kermode, 1998).

One of the earliest of these investigations (Henry et al, 1994), a cross-over study in nine patients to look at the effects of lavender on sleep patterns in dementia, showed an increased duration of sleep. This finding was supported by a later single case study (West \& Brockman, 1994) and by a study (Hardy et al, 1995) of four insomniac psychogeriatric patients with dementia, suggesting that ambient lavender oil was as effective in controlling poor sleep patterns as was their usual long-term drug treatment (temazepam, promazine or clomethiazole). Wolfe \& Herzberg (1996) also found evidence of improved sleep in one of two patients with severe dementia treated with lavender.

Another single-case design study (Brooker et al, 1997) allocated four patients with senile dementia to one of four interventions: ambient lavender, massage with lavender oil, massage without oil, and no treatment. They were exposed to these interventions over a 3-month period in 10 sessions, each lasting 30 minutes. The patient allocated to the therapy massage with lavender oil showed a significant improvement in behaviour in the hour after treatment.

MacMahon \& Kermode (1998) reported an individual case study of the effectiveness of aromatherapy massage with lavender oil on motivational behaviour in a dementia care setting using an AB design. Baseline data were recorded for 2 months, followed by 2 months of treatment data. Findings showed a statistically significant improvement in motivational behaviour associated with the use of aromatherapy. 
All of these studies share the problem of determining individual treatment effects when it is clear that there are often large individual differences in response. This would seem to argue for the implementation of larger placebo-controlled trials. However, some (Klemm et al, 1992) argue that it is these very differences that make the detailed study of individual responses to interventions more suitable than group designs.

\section{Placebo or case-control studies}

A small case-series study (Smallwood et al, 2001) undertook a randomised controlled trial of the relaxing effects of a lavender aromatherapy massage on disordered behaviour in dementia in 21 patients. Patients were randomly allocated into one of three conditions: aromatherapy and massage, aromatherapy and conversation, and massage only. Their behaviour was videotaped for a period of 1 hour (10.00-11.00 h, 11.00-12.00 h, 14.00-15.00 h, or 15.00-16.00 h) immediately after therapy (the duration of therapy was not reported). In blinded ratings of the video recordings, of the three conditions, aromatherapy and massage showed the greatest reduction in the frequency of excessive motor behaviour. However, this reached statistical significance $(P<0.05)$ only for the period 15.00-16.00 h.

A small placebo-controlled trial in which one of us (C.H.) was involved (Holmes et al, 2001) used masked observer ratings of agitated behaviour in 15 patients with severe dementia during lavenderstream aromatherapy (in which heated aromatherapy oil was dispersed into the atmosphere by a small fan) and during a placebo condition. During the aromatherapy sessions the observers detected improvement in nine patients $(60 \%)$, with a further five $(33 \%)$ showing no change and only one $(7 \%)$ showing a worsening of agitated behaviour. A comparison of the group median Pittsburgh Agitation Scale (PAS; Rosen et al, 1994) scores during aromatherapy showed a significant improvement in agitated behaviour during aromatherapy compared with placebo (median PAS scores 3 v. 4; Wilcoxon signed-ranks test $P=0.016$ (one-tailed)). Interestingly, although the numbers were small, there was a suggestion in this study that outcome of the intervention was diagnosis-specific, with patients who had dementia with Lewy bodies showing the least favourable effects. This study concluded that lavender oil administered in an aroma stream shows evidence of modest efficacy in the treatment of agitated behaviour in patients with severe dementia.

A videotaped study (Gray \& Clair, 2002), with high interrater reliability between masked observers, of 13 older people with dementia who were exposed to four aroma interventions during medication administration (lavender; sweet orange; tea tree; and no aroma (control)) failed to show any statistically significant differences between interventions for resistive behaviour.

Bowles et al (2002) conducted a cross-over study of aromatherapy massage in 56 patients with moderate to severe dementia. Creams containing one of four essential oils (lavender, sweet marjoram, patchouli or vetiver) or cream alone were massaged into the skin 5 times daily for 8 weeks. The study showed a significant decrease in behavioural problems and resistance to care in the patients who received the cream with essentials oils compared with those who received the cream alone.

The largest placebo-controlled study (Ballard et al, 2002), in which 72 patients with severe dementia were treated with lemon balm essential oil, demonstrated improvements in behavioural symptoms comparable with those seen with neuroleptic agents in patients with less severe dementia, but it also indicated secondary improvements in quality of life and activities. Half of the patients were randomly assigned to aromatherapy with lemon balm essential oil $(n=36)$ and half to placebo (sunflower oil). The active treatment oil or placebo oil was combined with a base lotion and applied to patients' faces and arms twice a day by care staff. Changes in clinically significant agitation (Cohen-Mansfield Agitation Inventory (CMAI) and quality of life indices (percentage of time spent socially withdrawn and percentage of time engaged in constructive activities, measured with Dementia Care Mapping) were compared between the two groups over a 4-week period of treatment. Sixty per cent of the active treatment group and $14 \%$ of the placebo-treated group experienced a $30 \%$ reduction in CMAI score, with an overall improvement in agitation (mean reduction in CMAI score) of $35 \%$ in patients receiving lemon balm essential oil and $11 \%$ in those treated with placebo (Mann-Whitney $U$ test; $Z=4.1, P<0.0001)$. Quality of life indices also improved significantly more in people receiving essential balm oil (Mann-Whitney $U$ test; percentage of time spent socially withdrawn: $Z=2.6$, $P=0.005$; percentage of time engaged in constructive activities: $Z=3.5, P=0.001)$. Importantly, at the end of the study no significant side-effects were observed. In this study, aromatherapy was used as an adjunct to existing psychotropic medication. Hence, although suggesting a place for aromatherapy as an adjunctive therapy, the study cannot be used as evidence that it is a viable alternative to sedative drugs in people with severe dementia. 


\section{Methodological issues}

There are several critical methodological issues to consider in trials using plant essential oils. As most people with severe dementia have lost any meaningful sense of smell, a direct placebo effect from a pleasant-smelling fragrance is an unlikely explanation for the positive effects of aromatherapy treatment. It is difficult to prevent raters (be they carers or research workers) from being able to identify the aroma of an active treatment, and it is therefore possible that a double-blind design may also be compromised. These pitfalls can be overcome to some degree by using observational measures as the primary outcomes, supplying researchers with nose clips or fragrant masks to wear when assessing participants, using a control fragrance with a detectable and pleasant odour or using masking smells such as air fresheners in the care environment.

There have also been large placebo responses in many studies examining the treatment of behavioural or psychiatric symptoms in people with dementia, and these are probably explained by the Hawthorne effect. It is therefore important in aromatherapy studies that the control intervention involves a similar amount of time and touch with the participant.

It is essential that these methodological issues are considered in the design of future studies investigating the potential role of aromatherapy in the clinical treatment of behavioural and psychiatric symptoms in people with dementia.

\section{Conclusions}

Observers' belief in aromatherapy makes studies of its efficacy essential. Although there is much casebased evidence suggesting its positive effect in improving sleep, agitated behaviours and resistance to care in dementia, there is a marked paucity of adequately sized studies that incorporate a placebocontrolled randomised element. One team (Ballard et al, 2002) has attempted this, with some positive results. However, their study still has its methodological flaws and it cannot determine whether aromatherapy offers a better alternative to existing pharmacological approaches.

The studies reviewed here raise important issues that need to be addressed. Most of the study samples included patients with different forms of dementia, even though it is clear that there are marked differences in treatment responses to other pharmacological agents between dementia subgroups. Indeed, one study (Holmes et al, 2001) suggests that patients who have dementia with Lewy bodies may show a deleterious response to aromatherapy. Although lavender and lemon balm are the oils most commonly used in the studies, they are delivered in a range of 'carriers', some involving the 'additional therapy' of physical contact with carers (e.g. skin creams, massage oils), others not (e.g. ambient aromatherapy streams), and in various treatment regimens. Clearly, any additional therapy such as massage needs to be minimised or controlled for before direct inferences can be made about aromatherapy alone. If we believe that there are active neurochemical differences between these agents then clearly a more rational approach would be based on the neurochemical profile of each agent, an area badly in need of research. Furthermore, research should profile not only the oils from different genuses but should also compare related species (e.g. Lavandula angustifolia and Lavandula officinalis).

To date, the widespread safe use of aromatherapies such as lavender tends to militate against any concerns about adverse side-effects. However, aromatherapy oil should not be seen as a safe alternative to existing pharmacotherapy until properly conducted safety trials have been completed. Indeed, it seems unlikely that the existence of neurochemical changes coupled with evidence of treatment effect in patients is associated with a complete absence of side-effects. It is clear that more well-designed, large-scale, randomised controlled trials are needed before conclusions can be drawn on the effectiveness of aromatherapy.

\section{References}

Aoshima, H. \& Hamamoto, K. (1999) Potentiation of GABA receptors expressed in Xenopus oocytes by perfume and phytoncid. Bioscience, Biotechnology and Biochemistry, 63, $743-748$

Ballard, C. G, O’Brien, J. T., Reichelt, K., et al (2002) Aromatherapy as a safe and effective treatment for the management of agitation in severe dementia: the results of a double-blind, placebo-controlled trial with Melissa. Journal of Clinical Psychiatry, 63, 553-558.

Bowles, E. J., Griffiths, D. M., Quirk, L., et al (2002) Effects of essential oils and touch on resistance to nursing care procedures and other dementia related behaviours in a residential care facility. International Journal of Aromatherapy, 12, 22-29.

Brooker, D. J. R., Snale, M., Johnson, E., et al (1997) Single case evaluation of the effects of aromatherapy and massage on disturbed behaviour in severe dementia. British Journal of Clinical Psychology, 36, 287-296.

Buchbauer, G., Jirovetz, L., Jager, W., et al (1993) Fragrance compounds and essential oils with sedative effects upon inhalation. Journal of Pharmaceutical Sciences, 82, 660-664.

Clark, D. L. \& Boutros, N. N. (1999) The Brain and Behaviour: An Introduction to Behavioural Neuroanatomy. Oxford: Blackwell Science.

Degel, J. \& Koster, E. P. (1999) Odors: implicit memory and performance effects. Chemical Senses, 24, 317-325.

Elisabetsky, E., Marschner, J. \& Souza, D. O. (1995) Effects of Linalool on glutamatergic system in the rat cerebral cortex. Neurochemical Research, 20, 461-465. 
Gray, S. G. \& Clair, A. A. (2002) Influence of aromatherapy on medication administration to residential-care residents with dementia and behavioural challenges. American Journal of Alzheimer's Disease and Other Dementias, 17, 169-174.

Hardy, M., Kirk-Smith, M. \& Stretch, D. (1995) Replacement of drug treatment for insomnia by ambient odour. Lancet 346, 701 .

Henry, J., Rusius, C. W., Davies, M., et al (1994) Lavender for night sedation of people with dementia. International Journal of Aromatherapy, 5 (2), 28-30.

Holmes, C., Hopkins, V., Hensford, C., et al (2001) Lavender oil as a treatment for agitated behaviour in severe dementia. International Journal of Psychogeriatric Psychiatry, 17, 305-308

Kite, S. M., Maher, E. J., Anderson, K., et al (1998) Development of an aromatherapy service at a cancer centre. Palliative Medicine, 12, 171-180.

Klemm, W. R., Lutes, S. D., Hendrix, D. V., et al (1992) Topographical EEG maps of human responses to odors. Chemical Senses, 17, 347-361.

Ludvigson, H. W. \& Rottmann, T. R. (1989) Effects of ambient odors of lavender and cloves on cognition, memory, affect and mood. Chemical Senses, 14, 525-536.

MacMahon, S. \& Kermode, S. (1998) A clinical trial of the effects of aromatherapy on motivational behaviour in a dementia care setting using a single subject design. Australian Journal of Holistic Nursing, 52, 47-49.

Price, S. \& Price, L. (1999) Aromatherapy for Health Professionals (2nd edn). London: Churchill Livingstone.

Rosen, J., Burgio, L., Kollar, M., et al (1994) The Pittsburgh Agitation Scale: a user friendly instrument for rating agitation in dementia patients. American Journal of Geriatric Psychiatry, 2, 52-59.

Smallwood, J., Brown, R., Coulter, F., et al (2001) Aromatherapy and behaviour disturbances in dementia: a randomized controlled trial. International Journal of Geriatric Psychiatry, 16, 1010-1013.

Vance, D. (1999) Considering olfactory stimulation for adults with age-related dementia. Perceptual and Motor Skills, $\mathbf{8 8}$ 398-400.

Wake, G., Court, J., Pickering, A., et al (2000) CNS acetylcholine receptor activity in European medicinal plants traditionally used to improve failing memory. Journal of Ethnopharmacology, 69, 105-114.

West, B. J. M. \& Brockman, S. J. (1994) The calming power of aromatherapy. Journal of Dementia Care, 2 (March/April) 20-22.

Wolfe, N. \& Herzberg, J. (1996) Can aromatherapy oils promote sleep in severely demented patients? International Journal of Geriatric Psychiatry, 11, 926-927.

Yamada, K., Mimaki, Y. \& Sashida, Y. (1994) Anticonvulsive effects of inhaling lavender oil vapour. Biological and Pharmaceutical Bulletin, 17, 359-360.

\section{Multiple choice questions}

1 The following plants have been used for psychiatric medical herbalism:

a lemon balm

b lavender

c chamomile

d valerian

e bergamot.

2 Linalool is:

a the main terpenoid component of lavender

b lipophilic

c associated with an increase in electroshock-induced convulsions in mice

$\mathrm{d}$ readily transported across the blood-brain barrier

e associated with augmentation of $\mathrm{GABA}_{\mathrm{A}}$.

3 In case studies of patients with dementia, lavender: a has been associated with improved sleep patterns

$\mathrm{b}$ has been associated with increased agitation

$c$ is more effective at high concentrations

$\mathrm{d}$ has been associated with improved motivational behaviour

e has been associated with anti-hallucinogenic effects.

4 The effects of aromatherapy in dementia:

a may be disease-specific

b may work independently of the ability to smell

c may be due to Hawthorne effects

d may differ according to the species of plant from which the oil is derived

e are independent of psychological aspects.

5 Difficulties in interpreting existing study data include:

a the paucity of randomised controlled trials

$b$ difficulty in incorporating an unidentifiable placebo

c variability in the use of adjunctive therapies

$\mathrm{d}$ the use of mixed populations of different dementia subtypes

e the lack of any likely neurochemical effects. 